\title{
A SIMILARITY PARAMETER FOR BREAKWATERS: THE MODIFIED IRIBARREN NUMBER
}

\author{
Maria Clavero, University of Granada, mclavero@ugr.es \\ Pedro Folgueras, University of Granada, folgueras@ugr.es \\ Pilar Diaz-Carrasco, University of Granada, pidiazc@ugr.es \\ Miguel Ortega-Sanchez, University of Granada, miguelos@ugr.es \\ Miguel A. Losada, University of Granada, mlosada@ugr.es
}

\section{INTRODUCTION AND JUSTIFICATION}

In the 14th ICCE, Battjes (1974) showed that a single similarity parameter only, embodying both the effects of slope angle and incident wave steepness, was important for many aspects of waves breaking on impermeable slopes, and suggested to call it the "Iribarren number", denoted by "Ir". Ahrens and McCartney (1975) verified the usefulness of Ir to describe run-up and stability on rough permeable slopes. Since then, many researchers applied Ir to characterize and to develop formulae for the design of breakwaters and to verify their stability.

On the other hand, depending on their typology, breakwaters reflect, dissipate, transmit, and radiate incident wave energy. Partial standing wave patterns are likely to occur at all types of breakwater, thus playing an important role in defining the wave regime in front of, near (seaward and leeward), and inside the breakwater. The characteristics of the porous medium, relative grain size $D / L$ and relative width, $A_{e q} / L^{2}$, are relevant magnitudes in that wave pattern (Vilchez et al. 2016), being $D$ the grain diameter, $L$ the wave length and $A_{\text {eq }}$ the porous area per unit section under the mean water level. $\mathrm{A}_{\mathrm{eq}} / \mathrm{L}^{2}$ is a scattering parameter controlling the averaged transformation of the wave inside the porous section of the structure. For a vertical porous breakwater (Type A), $A_{\text {eq }}$ is simply $B \cdot h$, and for a constant depth, the scattering parameter is reduced to $B / L$, which is the relative breakwater width.

\section{THE MODIFIED IRIBARREN NUMBER, $\mathrm{Ir}^{*}$}

Ir, as originally defined by Iribarren and Nogales (1949), is roughly proportional to the ratio of the tangent of the slope angle (the slope "steepness") to the local steepness of the breaking wave. Various physical interpretations of Ir can be given, but following Battjes (1974), its role from the viewpoint of model - prototype similarity is here revisited. Then, based on Dalrymple et al. (1991) and seeking for the possibility of full similarity, Ir is modified to introduce into the parameter the averaged transformation of the wave inside the porous section of the structure. Then, the slope "steepness" is substituted by the scattering parameter, obtaining the modified Iribarren number: $I r^{*}=\frac{A_{e q} / L^{2}}{\sqrt{H / L}}$

Several properties of the wave-breakwater interaction can be related with $\mid \mathrm{r}^{*}$. Figures 1 and 2 show the wave transformation (reflection and dissipation) and the stability of armour units versus Ir* $^{*}$ for RMB-CW (rubble mound with crown wall breakwater, Figure 3) for different armour types (cubes data from Gomez-Martin and Medina, 2014). It should be noticed that it is common the core and the secondary layers of breakwaters in the lab to be distorted. Then, the froudian model-prototype similarity might be achieved provided that $\mathrm{Ir}^{*}$ is the same in the model and the prototype.

\section{CONCLUSIONS}

The modified Iribarren number is (1) a good indicator of many overall properties involved in the design and performance of breakwaters, and (2) helps to approach to the possibility of full similarity under wave reflectivebreaking conditions.
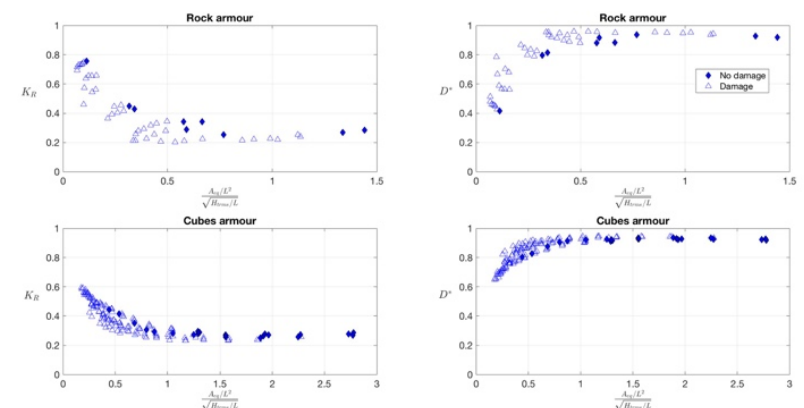

Figure 1 - Reflection and dissipation versus the modified Iribarren number for a RMB-CW breakwater
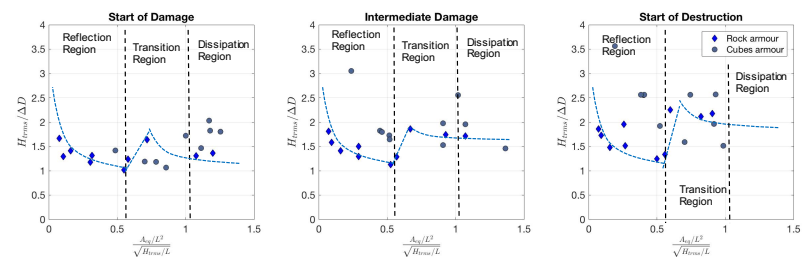

Figure 2 - Stability function versus the modified Iribarren number for a RMB-CW breakwater

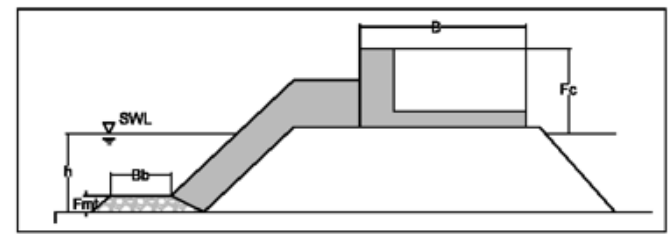

Figure 3 - RMB-CW breakwater idealized cross-section

\section{REFERENCES}

Ahrens and McCartney (1975): Wave period effect on the stability of rip-rap, ASCE, PCEng. in the Oceans/III, Vol.2, pp. 1019-1034.

Battjes (1974): Surf Similarity, ICCE, pp. 466-480.

Dalrymple, Losada and Martin (1991): Reflection and transmission from porous structures under oblique wave attack, J. Fluid Mech. 224, pp. 625-644.

Gomez-Martin and Medina (2014). Heterogeneous Packing and Hydraulic Stability of Cube and Cubipod Armor Units. J. Waterway, Port, Coastal, Ocean Eng., 140(1): 100-108

Iribarren and Nogales (1949): Protection des Ports, Section II, XVIIth Int. Nav. Cong., Lisbon, pp. 31-80.

Vilchez, Clavero and Losada (2016): Hydraulic perfomance of different non-overtopped breakwater types under 2D wave attack, CENG, 107, pp. 34-52. 